\title{
-Original-
}

\section{The clinical significance of posterior gastric vein in portal hypertension as visualized in portograms}

\author{
Liu Yong Feng ${ }^{1 * *}$, Zheng Xiang Dong ${ }^{1) *}$, Takashi Tajiri ${ }^{1)}$, \\ Matsuomi Umehara ${ }^{1}$, Kiyohiko Yamashita ${ }^{1}$, Masahiko Onda' ${ }^{11}$, \\ Tohru Ohya ${ }^{2)}$, Seiichi $\mathrm{Hosoi}^{2)}$ and Tatsuo Kumazaki²) \\ 1) The First Department of Surgery (Chief: Prof. Masahiko Onda), \\ Nippon Medical School, Tokyo, Japan \\ 2) Department of Radiology, Nippon Medical School, Tokyo, Japan
}

\begin{abstract}
Summary
The posterior gastric vein was detected angiographically in 94 of 110 patients with portal hypertension (85.5\%). The surgical importance of the posterior gastric vein derives from its relatively high incidence, from its being another source of reverse blood flow to the varices, from its having an almost hidden origin from the posterior gastric wall and from the lack of attention to the vessel. The clinical significance of the posterior gastric vein in portal hypertension is emphasized in this article.
\end{abstract}

Key words: posterior gastric vein, portal hypertension, portography, angiography, divascularization

\section{Introduction}

The posterior gastric vein is one of the tributaries of the portal system. It dilates in portal hypertension and is one of the sources of the reversal of blood flow into the gastric fundus and esophagus. However, this fact have been ignored or neglected for centuries, and there has been no description of this in most modern textbooks of anatomy and surgery. An anatomic study of 62 cadavers was reported in China in $1982^{11}$. The author proposed that the posterior gastric vein is an important blood vessel which causes bleeding in portal hypertension. Since then, more attention has been directed toward this vein in the operations of portal hypertension in China. We analysed the portograms of 110 patients with portal hypertension to observe the incidence of the posterior gastric vein and the changes of the vessel in abnormal condition of portal system.

\section{Materials and Methods}

The portograms of 110 patients with portal hypertension, performed at the departments of surgery and radiology of Nippon Medical School, were studied retrospectively. Of these patients, 81 were male and 29 female. Ages ranged from 17 to 78 years ( $50.9 \pm 0.9$ years). Eighty-five of 110 patients had a history of hemorrhage $(77.3 \pm 4.0 \%)$. The degree of hepatic function according to Child's classification was A degree 13, B 43, and C 54 cases. 
Portograms were taken by the technique of the percutaneous transhepatic portography (PTP), or the transileocolic vein portography (TIP) ${ }^{2)}$. Surgimed ${ }^{\circledR}$ (Denmark) $6.6 \mathrm{~F}$ and $\mathrm{Cook}^{\circledR}$ (USA) $6.5 \mathrm{~F}$ catheters were used for the catheterization of the splenic vein. The contrast medium used was $76 \%$ meglumine diatrizoate and a single injection of $40 \mathrm{~m} l$ was administered into the splenic vein at a rate of $8 \sim 10 \mathrm{~m} l$ per second. The programing was done with 2 frames/second for 3 seconds, followed by 1 frame/second for 3 seconds, and then 1 frame every 2 seconds for a total of 18 seconds. The film size exposured was 14 by 14 inches in all cases.

\section{Results}

The posterior gastric vein was detected angiographically in 94 out of 110 patients $(85.5 \pm$ $3.5 \%)$ and absent in the remaining $16(14.5 \pm 3.4 \%)$. The single posterior gastric vein was found in 84 cases $(89.4 \pm 3.2 \%)$ (Fig. 1), double in 10 cases $(10.6 \pm 3.2 \%)$ (Fig. 2). The posterior gastric vein drained into the splenic vein at the mid-third in 78 cases $(83.0 \pm 3.8 \%)$, while in 16 cases $(17.0 \pm$ $3.9 \%$ ) at the distal-third to the spleen. According to the degree of dilatation of the posterior gastric

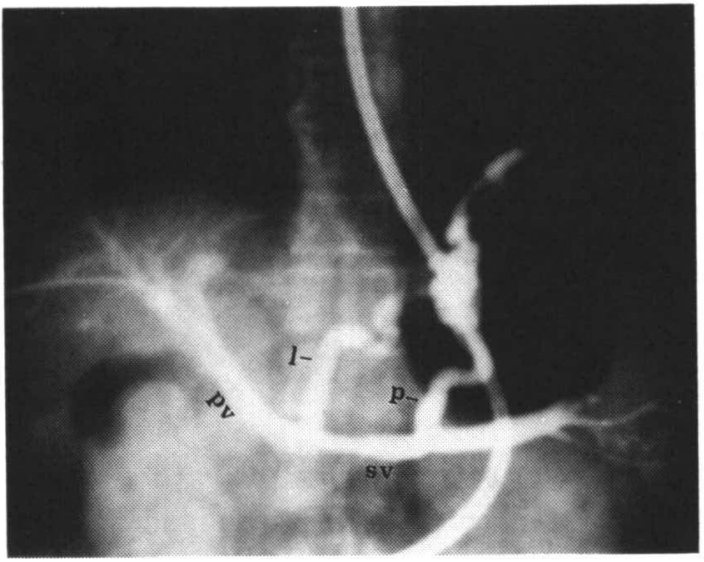

Fig. 1 PTP portogram shows the posterior gastric vein $(\mathrm{P})$, the left gastric vein $(\mathrm{L})$, the portal vein $(\mathrm{PV})$ and the splenic vein (SV)

The posterior gastric vein is a single vessel. It is a tributary of the splenic vein. The dilatation of the posterior and the left gastric vein is almost the same in diameter.

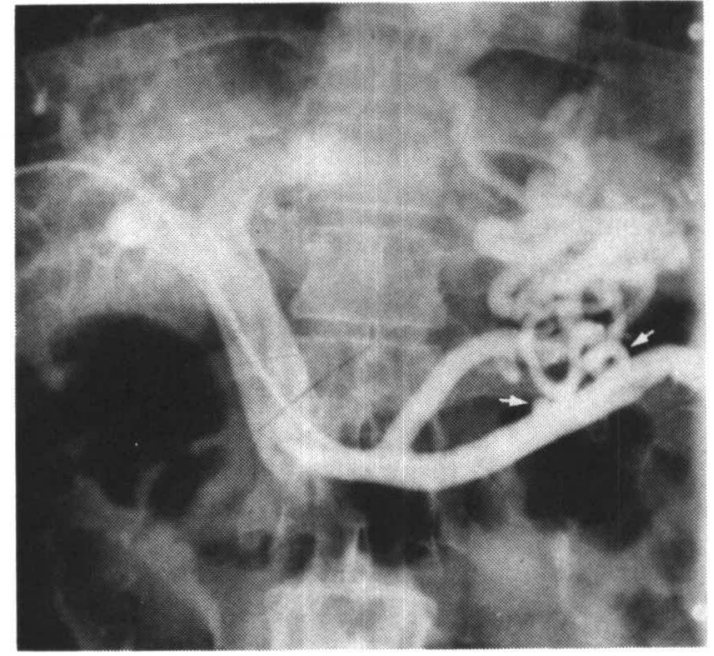

Fig. 2 PTP portogram shows the double posterior gastric vein, indicated by the two arrows

Table 1 The dilatation type and the bleeding rate of the posterior and left gastric vein

\begin{tabular}{c|cccc}
\hline Type & $\begin{array}{c}\text { No. of } \\
\text { patients }\end{array}$ & Rate & $\begin{array}{c}\text { No. of } \\
\text { bleeding }\end{array}$ & Rate \\
\hline $\begin{array}{c}\text { A. posterior gastric } \\
\text { vein mainly }\end{array}$ & 21 & $19.1 \pm 3.7 \%$ & 16 & $76.2 \pm 9.3 \%$ \\
$\begin{array}{c}\text { B. equal dilatation } \\
\text { of posterior and } \\
\text { left gastric vein }\end{array}$ & 20 & $18.2 \pm 4.2 \%$ & 16 & $80.0 \pm 8.9 \%$ \\
$\begin{array}{c}\text { C. left gastric } \\
\text { vein mainly }\end{array}$ & 69 & $62.7 \pm 4.6 \%$ & 53 & $76.8 \pm 5.1 \%$ \\
\hline Total & 110 & $100 \%$ & 85 & $77.3 \pm 4.0 \%$ \\
\hline
\end{tabular}




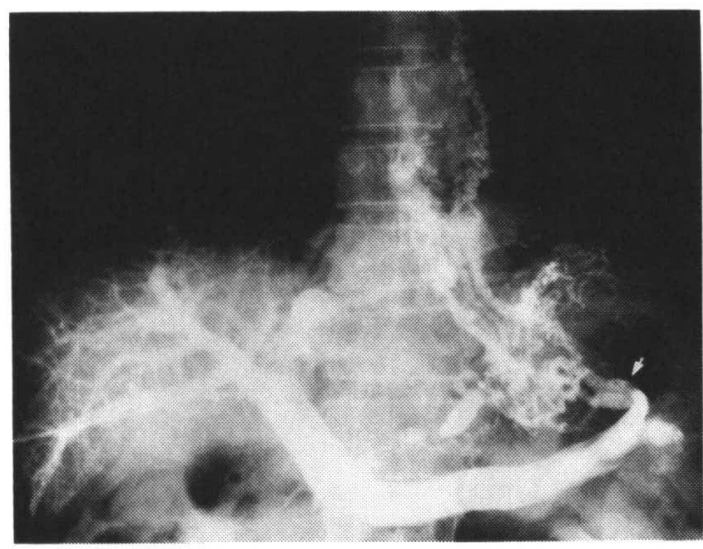

Fig. 3 PTP portogram shows that the posterior gastric vein is the main dilated vessel, indicated by the arrow

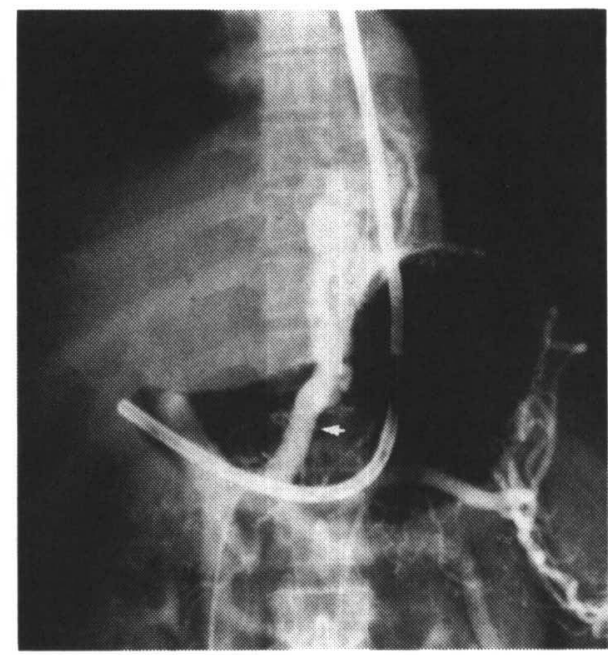

Fig. 4 TIP portogram shows that the left gastric vein is the main dilated vessel, indicated by the arrow

vein, as well as the proportion of dilation to that of the left gastric vein and connection with the varices, we divided the 110 patients into three types (Table 1): (A). dilatation mainly of posterior gastric vein occurred in 21 patients $(19.1 \pm 3.7 \%$ ) (Fig. 3); (B). equal dilatation of the posterior and left gastric vein in 20 patients $(18.2 \pm 4.2 \%$ ) (Fig. 1); (C). dilatation mainly of the left gastric vein (including 16 patients without the posterior gastric vein) in 69 cases $(62.7 \pm 4.6 \%)$ (Fig. 4). The bleeding rate was $76.2 \pm 9.3 \%(16: 21)$ in type A, $80.0 \pm 8.9 \%(16: 20)$ in type B, $76.8 \pm 5.1 \%(53: 69)$ in type $\mathrm{C}$ (Table $\mathbf{1})$.

\section{Discussion}

The posterior gastric vein commences from small tributaries which are distributed on the posterior wall of the abdominal esophagus and posterior wall of the superior portion of the gastric body, near the cardiac region, and the fundus. These unite into a single vessel $(89.4 \pm 3.2 \%)$ or a double $(10.6 \pm 3.2 \%)$, which is accompanied by the posterior gastric artery ${ }^{1,3,4)}$, running behind the parietal peritonum of the omental burser, entering the splenic vein at the mid-third $(83.0 \pm 3.8 \%)$ or distal-third $(17.0 \pm 3.9 \%)$. The posterior gastric vein and artery produces a peritoneal fold before leaving and reaching the posterior wall of the stomach, which is a mark for surgeons to find this vessel during operations.

The posterior gastric vein belongs to the portal system but it is not as regular as other tributaries. In some people it may be absent. The incidence of posterior gastric veins was $80.6 \%$ according to Liu in $1982^{1)}$ in China using the anatomic method. In this study the incidence was $85.5 \%$. The results show that there is no significant difference between Japanese and Chinese. The posterior gastric vein was detected in 94 out of 110 patients, in whom the vein was always dilated in a different degree.

According to the dilated degree of the posterior gastric vein and proportion of dilation to that of the left gastric vein, in connection with the varices, we divided the 110 patients into three types as mentioned above. From the view point of dilated frequence of left and posterior gastric veins, 


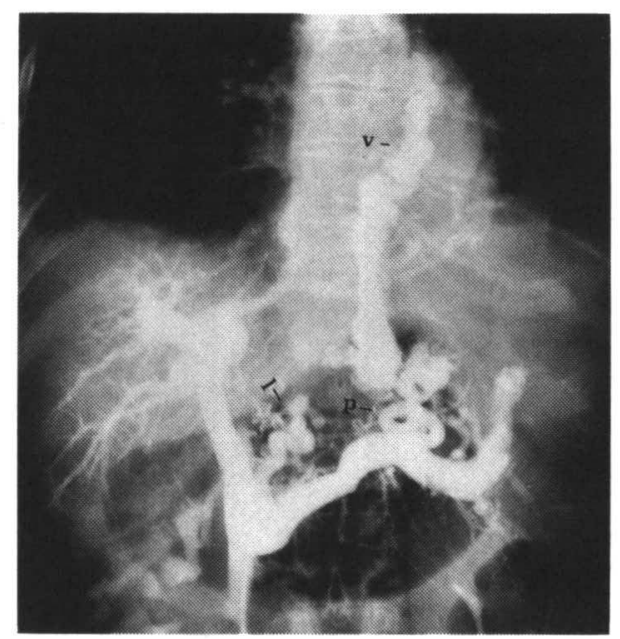

Fig. 5 TIP portogram shows that after the left gastric vein was embolized $(\mathrm{L})$ the varices $(\mathrm{V})$ did not disappear, and blood could still regurgitate from the posterior gastric vein $(\mathrm{P})$ to the esophageal varices

this study suggests that the left gastric vein is very important in portal hypertension, because of both its dilated frequence and the hemorrhage rate $(76.8 \pm 5.1 \%)$, slightly higher than the $60.0 \%$ found by Inokuchi and others ${ }^{5}$. However, the posterior gastric vein could not be disregarded because in $19.1 \pm 3.7 \%(21: 110)$ patients the prominent dilatation of the vessel with a bleeding rate of $76.2 \pm 9.3 \%$ (16:21) was documented, and in $18.2 \pm 4.2 \%(20: 110)$ patients, the dilatation of the posterior gastric vein was the same as that of the left gastric vein with a bleeding rate of 80.0 $\pm 8.9 \%(16: 20)$. Therefore, the posterior gastric vein is also very important. From Fig. 5 we can see that after the embolization of the left gastric vein the varices did not disappear, and blood could still regurgitate from the posterior gastric vein to the esophageal varices. Thus, for devascularization or embolization the posterior gastric vein should, if it exists, be ligated or embolized to avoid dilatating and rebleeding due to the higher pressure of the portal system. Had the posterior gastric vein been left out, it could be one of the causes of rebleeding after devascularization or embolization.

\section{References}

1) Liu, Y.-F. and Xu, E.-D.: Regional anatomy of the posterior gastric artery and vein and its importance in surgery. Chin. J. Surg., 20, 520 525, 1982.

2) Tajiri, T., Miki, M. and Shirota, A.: Clinical significance of direct portography in portal hypertension and surgery of the hepatobiliary tract. Thai. J. Surg., 5, 45 52, 1984.

3) Suzuki, K., Prates, J.C. and Didio, L.J.A.: Incidence and surgical importance of the posterior gastric artery. Ann. Surg., 187, 134 136, 1978.

4) Didio, LJ.A., Christoforidis, A. and Chandnani, P.C.: Posterior gastric artery and its significance as seen in angiograms. Am. J. Surg., 139, 333 337, 1980.

5) Inokuchi, K., Kobayashi, M., Kusaba, A., Ogawa, Y., Saku, M. and Shiizaki, T.: New selective decompression of esophageal varices. Arch. Surg., 100, 157 162, 1970. 